\title{
Dose-dependent effect of daptomycin on the artificial prolongation of prothrombin time in coagulation abnormalities: in vitro verification
}

\author{
Hideki Hashimoto ${ }^{1,2}$, Makoto Saito ${ }^{1}$, Naoki Kanda², Takehito Yamamoto ${ }^{3}$, Makiko Mieno $^{4}$ and Shuji Hatakeyama ${ }^{2,5^{*}}$
}

\begin{abstract}
Background: Several studies have reported that daptomycin induced artificial prolongation of prothrombin time (PT) in some test reagents, particularly in warfarin users. However, it remains unknown whether the artificial prolongation can be affected by coagulation abnormalities other than the use of warfarin. Thus, we investigated the effect of daptomycin on PT with two types of coagulation abnormalities.

Methods: Plasma samples were pooled by four groups: healthy volunteers (Plasma A), warfarin users with a PT-international normalized ratio (INR) of approximately 2.0 (Plasma B) or 3.0 (Plasma C), and patients with liver cirrhosis with a PT-INR of approximately 2.0 (Plasma D). Plasma A was composed of plasma from two healthy individuals ( $9 \mathrm{~mL}$ from each individual). Plasma B, C, and D were composed of plasma from 36 patients $(0.5 \mathrm{~mL}$ from each patient). Daptomycin was added to each sample to create solutions with several concentrations (0-150 $\mu \mathrm{g} / \mathrm{mL})$. The PT-INR for each solution was measured with three PT reagents. Linear regression analyses were used to determine the association between daptomycin concentration and PT-INR. The relative change in PT-INR due to daptomycin concentrations was calculated.
\end{abstract}

Results: Strong linear correlations were observed between daptomycin concentrations and PT-INR for all the plasma groups and reagents $\left(R^{2}>0.7, P<0.01\right)$. At a daptomycin concentration of $150 \mu \mathrm{g} / \mathrm{mL}$, the relative increase of PT-INR was $\geq 10 \%$ in the majority of the plasma groups with an elevated baseline PT-INR in all reagents tested.

Conclusions: Daptomycin induced the artificial prolongation of PT-INR in a concentration-dependent manner, particularly in plasma samples with an elevated baseline PT-INR. PT should be evaluated at the trough levels of daptomycin.

Keywords: Daptomycin, Prothrombin time, Coagulation abnormality, Warfarin, Liver cirrhosis, False positive

\section{Background}

Daptomycin is a cyclic lipopeptide antibiotic that is used to treat Gram-positive bacteria, such as methicillinresistant Staphylococcus aureus (MRSA) [1]. It is inserted into the cytoplasmic membrane of Grampositive bacteria and then disrupts the membrane,

\footnotetext{
* Correspondence: shatake-tky@umin.ac.jp

${ }^{2}$ Division of General Internal Medicine, Jichi Medical University Hospital,

3311-1 Yakushiji, Shimotsuke-shi, Tochigi 329-0498, Japan

${ }^{5}$ Division of Infectious Diseases, Jichi Medical University Hospital, 3311-1

Yakushiji, Shimotsuke-shi, Tochigi 329-0498, Japan

Full list of author information is available at the end of the article
}

causing bacterial death. Daptomycin was approved by the United States Food and Drug Administration (FDA) in 2003 for the treatment of complicated skin and soft tissue infections and $S$. aureus bloodstream infections. Currently, it is also recommended for many serious infections caused by Gram-positive bacteria [2].

Some studies have reported the prolongation of prothrombin time (PT) in daptomycin users [3-5]. This prolongation is considered to be artificial because patients did not have actual bleeding tendencies, and it was observed when specific recombinant thromboplastin 
reagents were used to examine PT [3]. PT prolongation was concentration dependent, and therefore, the FDA suggests that PT should be evaluated at the trough levels of daptomycin [6].

Since this prolongation was not previously evaluated clinically, we did a prospective in vivo study to determine the effect of the daptomycin-induced PT prolongation in clinical settings using nine PT reagents that are available in Japan. Using several specific reagents, we confirmed that PT was significantly prolonged when daptomycin concentrations were high, particularly in warfarin users [7]. However, in vitro studies in a more controlled condition should be conducted to verify the results of our in vivo study. In addition, it remains unknown whether this prolongation is similarly observed in patients with other coagulation disorders, such as liver cirrhosis. Therefore, we conducted an in vitro study to determine whether daptomycin affects the PTinternational normalized ratio (INR) in patients with coagulation abnormalities in addition to warfarin users.

\section{Methods}

\section{Study population and setting}

This study was based on pooled plasma samples from four groups: healthy volunteers (Plasma A), patients who took warfarin and whose PT-INR values were approximately 2.0 (Plasma B) or 3.0 (Plasma C), and patients who had liver cirrhosis and whose PT-INR values were approximately 2.0 (Plasma D). Patients who had liver cirrhosis and took warfarin were excluded in addition to patients with antiphospholipid syndrome. Plasma from two healthy individuals' plasma was used for Plasma A. Plasma B, C, and D consisted of 36 patients' plasma each. All patients were outpatients who visited the Jichi Medical University Hospital between June 2016 and August 2016.

\section{Sample preparation}

Plasma samples were collected from 108 patients (0.5 mL each) and two healthy individuals $(9 \mathrm{~mL}$ each) and stored at $-80{ }^{\circ} \mathrm{C}$. After all the samples were collected, frozen plasma samples were thawed and pooled by group. Daptomycin was added to each group of plasma samples to obtain $0,5,15,50,100$, and $150 \mu \mathrm{g} /$ $\mathrm{mL}$ solutions. Daptomycin was purchased from Merck Sharp \& Dohme (Tokyo, Japan).

We examined the PT-INR of these solutions using three reagents. Reagent 1 (HemosIL RecombiPlasTin 2G: a recombinant human thromboplastin) was purchased from Werfen Japan (Tokyo, Japan). Reagent 2 (Neoplastin plus: a rabbit brain thromboplastin) and Reagent 3 (STA Neoplastin R: a recombinant human thromboplastin) were purchased from Roche Diagnostics
K. K. (Tokyo, Japan). The international sensitivity index for each reagent was $0.97,1.29$, and 0.94 , respectively.

\section{Data collection and statistical analyses}

The PT-INR of all plasma samples were measured three times. The mean and standard deviation (SD) were calculated. Linear regression analyses for each plasma group and reagent were used to assess the associations between daptomycin concentrations and PT-INR. $P$ values less than 0.05 were considered statistically significant. Statistical analyses were performed using JMP Pro 12 (SAS Institute, Cary, North Carolina, USA). In addition, we calculated the relative change in PT-INR between high daptomycin concentrations (50, 100, and $150 \mu \mathrm{g} / \mathrm{mL})$ versus the control $(0 \mu \mathrm{g} / \mathrm{mL})$ in each plasma group. We regarded $10 \%$ of the relative change in PTINR as clinically significant, as described in previous studies [3, 7].

\section{Results}

We found strong and significant linear correlations between daptomycin concentrations and PT-INR for all the plasma groups and reagents $\left(R^{2}>0.7, P<0.01\right.$ for all the groups and reagents) (Table 1 and Fig. 1). When the daptomycin concentration was $100 \mu \mathrm{g} / \mathrm{mL}$, the PT-INR was prolonged by more than $10 \%$ in Plasma $\mathrm{C}$ using Reagent 1 and Plasma B, C, and D, respectively, using Reagent 3 (Table 2). At a daptomycin concentration of $150 \mu \mathrm{g} / \mathrm{mL}$, almost all the relative changes in PT-INR in Plasma B, C, and D were more than $10 \%$ for all reagents. The maximum PT-INR prolongation was $22 \%$ when the daptomycin concentration was $150 \mu \mathrm{g} / \mathrm{mL}$ in Plasma D using Reagent 3 (Table 2).

Table 1 Results of the linear regression analysis of daptomycin concentrations and PT-INR

\begin{tabular}{llllll}
\hline Reagent & Plasma group & $R^{2}$ & $\beta$ & $S E(\beta)$ & $P$-value \\
\hline 1: HemosIL RecombiPlasTin 2G & A (INR 1) & 0.74 & $2.2 \times 10^{-4}$ & $3.3 \times 10^{-5}$ & $<0.0001$ \\
& B (warfarin, INR & 0.97 & $1.5 \times 10^{-3}$ & $6.0 \times 10^{-5}$ & $<0.0001$ \\
2) & & & & \\
& C (warfarin, INR & 0.94 & $2.5 \times 10^{-3}$ & $1.5 \times 10^{-4}$ & $<0.0001$ \\
3) & & & & \\
D (LC, INR 2) & 0.96 & $1.3 \times 10^{-3}$ & $6.5 \times 10^{-5}$ & $<0.0001$ \\
2: Neoplastin Plus & A (INR 1) & 0.76 & $5.1 \times 10^{-4}$ & $7.3 \times 10^{-5}$ & $<0.0001$ \\
& B (warfarin, INR & 0.75 & $8.6 \times 10^{-4}$ & $1.2 \times 10^{-4}$ & $<0.0001$ \\
2) & & & & \\
C (warfarin, INR & 0.94 & $1.9 \times 10^{-3}$ & $1.3 \times 10^{-4}$ & $<0.0001$ \\
3) & & & & \\
D (LC, INR 2) & 0.89 & $1.4 \times 10^{-3}$ & $1.2 \times 10^{-4}$ & $<0.0001$ \\
A (INR 1) & 0.88 & $6.6 \times 10^{-4}$ & $6.0 \times 10^{-5}$ & $<0.0001$ \\
3: STA Neoplastin R & B (warfarin, INR & 0.95 & $2.4 \times 10^{-3}$ & $1.3 \times 10^{-4}$ & $<0.0001$ \\
& 2) & & & & \\
C (warfarin, INR & 0.97 & $3.3 \times 10^{-3}$ & $1.6 \times 10^{-4}$ & $<0.0001$ \\
3) & & & & \\
& D (LC, INR 2) & 0.99 & $2.2 \times 10^{-3}$ & $4.7 \times 10^{-5}$ & $<0.0001$ \\
\hline & & & & &
\end{tabular}

INR international normalized ratio, SE standard error, LC liver cirrhosis 


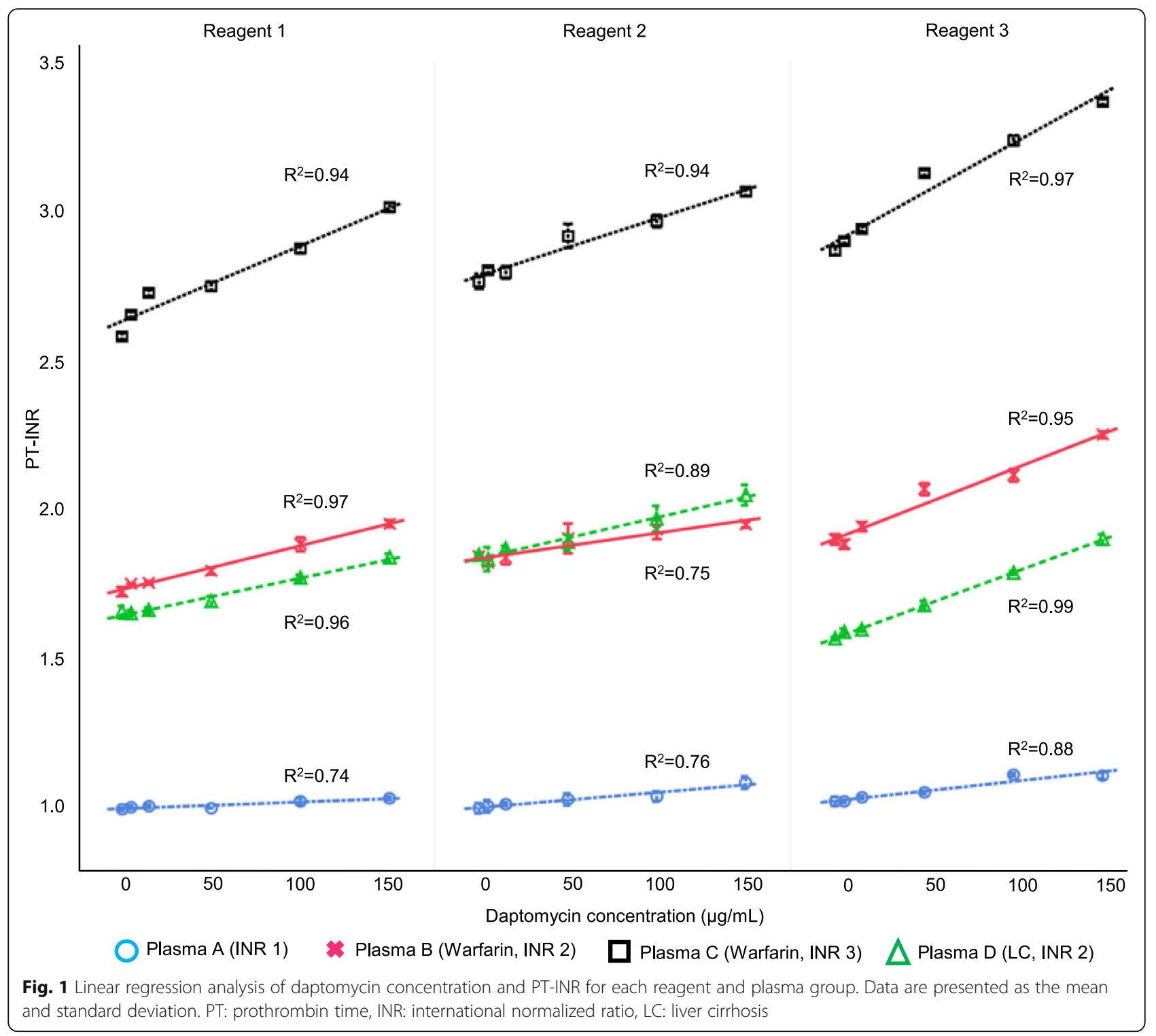

For Reagents 1 and 3, the relative increase in PT-INR appeared to be greater in the plasma groups with a high baseline PT-INR (Plasma B, C, and D) than in Plasma A with a normal baseline PT-INR (Table 2). On the other hand, there were no apparent differences in the relative changes in PT-INR between Plasma B and D, which had comparable PT-INR levels at baseline. For Reagent 2, there were no obvious differences between the plasma groups with a high baseline PT-INR (Plasma B, C, and $\mathrm{D})$ and that with a normal baseline PT-INR (Plasma A): all groups responded to the similar extent.

\section{Discussion}

We found a strong and significant linear association between daptomycin concentrations and the PT-INR prolongation in all the plasma groups and reagents. When the daptomycin concentration was higher than $100 \mu \mathrm{g} /$ $\mathrm{mL}, \mathrm{PT}$-INR was prolonged by approximately $10-20 \%$, particularly in those with elevated baseline PT-INR. These results are consistent with the findings in our previous in vivo study [7]. In our previous study, the linear association was observed only in Reagents 2 and 3 . However, this result might be due to other clinical factors that can affect the vital coagulation system. Moreover, plasma samples with a high concentration (e.g., > $100 \mu \mathrm{g} / \mathrm{mL}$ ) were limited in our previous study. Not all reagents used in this study have been tested in previous in vitro studies. In the present study, we confirmed the daptomycin concentration-dependent prolongation of PT-INR under controlled conditions.

In our previous study, peak daptomycin concentrations ranged from 30 to $90 \mu \mathrm{g} / \mathrm{mL}$ after the administration of 
Table 2 Baseline PT-INR values and ratios of PT-INR to the baseline PT- INR at designated daptomycin concentrations

\begin{tabular}{|c|c|c|c|c|c|}
\hline Reagent & Plasma group & $\begin{array}{l}\text { Baseline PT-INR } \\
\text { (mean } \pm \text { SD) }\end{array}$ & $\begin{array}{l}\text { PT- } \\
\text { INR }\end{array}$ & $\begin{array}{l}\text { PT- } \\
\text { INR } \\
\text { PT-INR } \\
\text { PT }\end{array}$ & $\begin{array}{l}\text { PT- } \\
\text { INR }_{150} \\
\text { PT-INR }_{0}^{\text {In }}\end{array}$ \\
\hline \multirow{4}{*}{$\begin{array}{l}\text { 1: HemoslL } \\
\text { RecombiPlasTin } \\
2 \mathrm{G}\end{array}$} & $A($ INR 1) & $0.96 \pm 0.00$ & 1.01 & 1.03 & 1.04 \\
\hline & B (warfarin, INR 2) & $1.70 \pm 0.01$ & 1.04 & 1.09 & 1.14 \\
\hline & $C$ (warfarin, INR 3) & $2.57 \pm 0.01$ & 1.07 & 1.11 & 1.17 \\
\hline & $\mathrm{D}(\mathrm{LC}, \mathrm{INR} 2)$ & $1.63 \pm 0.02$ & 1.02 & 1.07 & 1.11 \\
\hline \multirow{4}{*}{$\begin{array}{l}\text { 2: Neoplastin } \\
\text { Plus }\end{array}$} & $A(I N R$ 1) & $0.97 \pm 0.02$ & 1.03 & 1.05 & 1.09 \\
\hline & B (warfarin, INR 2) & $1.82 \pm 0.01$ & 1.03 & 1.05 & 1.06 \\
\hline & $C$ (warfarin, INR 3) & $2.75 \pm 0.02$ & 1.06 & 1.07 & 1.11 \\
\hline & $\mathrm{D}(\mathrm{LC}, \mathrm{INR} 2)$ & $1.83 \pm 0.00$ & 1.02 & 1.07 & 1.11 \\
\hline \multirow{4}{*}{$\begin{array}{l}\text { 3: STA } \\
\text { Neoplastin R }\end{array}$} & A (INR 1) & $0.99 \pm 0.02$ & 1.03 & 1.09 & 1.09 \\
\hline & B (warfarin, INR 2) & $1.88 \pm 0.02$ & 1.09 & 1.12 & 1.19 \\
\hline & $C$ (warfarin, INR 3) & $2.86 \pm 0.02$ & 1.09 & 1.13 & 1.18 \\
\hline & $\mathrm{D}(\mathrm{LC}, \mathrm{INR} 2)$ & $1.54 \pm 0.01$ & 1.07 & 1.14 & 1.22 \\
\hline
\end{tabular}

PT prothrombin time, INR international normalized ratio, SD standard deviation, LC liver cirrhosis

${ }^{a}$ PT-INR 0 represents the baseline PT-INR. PT-INR ${ }_{50}$, PT-INR 100 , and PT-INR ${ }_{150}$ represent the PT-INR at daptomycin concentrations of 50,100 , and $150 \mu \mathrm{g} / \mathrm{mL}$, respectively. The relative change in PT-INR was calculated using the mean values of PT-INR measured in triplicate at each daptomycin concentration

$6 \mathrm{mg} / \mathrm{kg}$ of daptomycin [7]. Another study showed that daptomycin doses of more than $6 \mathrm{mg} / \mathrm{kg}$ led to higher peak daptomycin concentrations of more than $100 \mu \mathrm{g} / \mathrm{mL}$ [8]. Currently, the high doses of daptomycin are sometimes recommended for the treatment of serious infections. For example, $10 \mathrm{mg} / \mathrm{kg}$ of daptomycin is recommended for the treatment of persistent MRSA bacteremia [2]. We must take into consideration that severe infections caused by Gram-positive pathogens frequently occur in warfarin users because some of them have intra-cardiac devices, such as ventricular assist devices or prosthetic heart valves. Patients with intra-cardiac devices have higher PT-INR levels (up to 3.5) $[9,10]$. If a serious infection caused by Gram-positive bacteria occurs in such patients, high doses of daptomycin will be administered, leading to a peak daptomycin concentration of more than $100 \mu \mathrm{g} / \mathrm{mL}$. Consequently, PT-INR may be artificially prolonged by more than $10 \%$, consequentially leading to an inappropriate reduction of the warfarin dose. We should ensure that PT is checked at the trough time of daptomycin concentrations.

For Reagents 1 and 3, the relative increase in PT-INR was higher in the plasma groups with a high baseline PT-INR (Plasma B, C, and D) than in the plasma group with a normal baseline PT-INR (Plasma A). The etiology of the coagulation abnormality also did not affect the relative change in PT-INR in all three reagents. These results can be explained by the fact that daptomycin interferes with phospholipids contained in the reagents rather than the coagulation factors in the plasma, which are decreased in both warfarin users and patients with liver cirrhosis. Although Webster et al. reported that prolonged baseline PT itself did not affect the relative change in PT, they lacked data on the relative change in PT in anticoagulated plasma samples with more than $100 \mu \mathrm{g} / \mathrm{mL}$ of daptomycin [3]. Our findings suggest that with high baseline PT-INR, it becomes more likely to observe clinically important relative change of $>10 \%$ in PT-INR in at least some reagents.

Previous studies have shown that two factors in PT reagents may be responsible for the false PT prolongation: the thromboplastin source and the phospholipid type of reagents $[3,4]$. It is conceivable that daptomycin that is inserted into the phospholipid component of PT reagents can interfere with tissue factor-induced coagulation, resulting in false PT prolongation [3]. Reagents made from recombinant human or rabbit tissue factors were highly susceptible to daptomycin when phosphatidylglycerol is added [4]. In this study, Reagents 1 and 3 were made from recombinant human tissue factors, although Reagent 2 was made from the rabbit brain. Therefore, the differences in the thromboplastin source might affect the susceptibility to daptomycin.

Our study had several limitations. First, we did not conduct statistical comparisons of the relative changes in PT-INR between the plasma groups due to the small sample sizes. In addition, biochemical studies are required to assess the mechanism by which daptomycininduced artificial prolongation was larger in the plasma with elevated baseline PT-INR levels than in the normal plasma, regardless of the cause.

\section{Conclusions}

In summary, we found that daptomycin induced the dose-dependent artificial prolongation of PT-INR in individuals with or without coagulation abnormalities. For some reagents, the extent of the relative change in PTINR was higher in the plasma groups with a higher baseline PT-INR due to either warfarin or liver cirrhosis than in the plasma group with a lower PT-INR. The etiology of coagulation abnormalities did not apparently affect the relative change. The evaluation of PT-INR at the trough time of daptomycin must be considered, particularly in patients with a higher baseline PT-INR.

\section{Abbreviations}

FDA: Food and drug administration; INR: International normalized ratio; LC: Liver cirrhosis; MRSA: Methicillin-resistant Staphylococcus aureus; PT: Prothrombin time; SD: Standard deviations; SE: Standard error

\section{Acknowledgements}

We would like to acknowledge all of the patients involved in this study. We would also like to thank Takako Muto from the Center for Community Medicine of Jichi Medical University for collecting and preserving the serum samples. We express our gratitude to the following companies for the technical support during PT measurements: Werfen Japan (Tokyo, Japan) and Roche Diagnostics K. K. (Tokyo, Japan).

Funding

The authors have no funding to declare. 


\section{Availability of data and materials}

All data generated or analyzed during this study are included in the published article.

\section{Authors' contributions}

SH designed and supervised the study. $\mathrm{HH}, \mathrm{NK}$, and TY obtained the data. $\mathrm{HH}, \mathrm{MS}, \mathrm{MM}$, and SH analyzed and interpreted the data. $\mathrm{HH}$ wrote the manuscript. MS and SH revised the manuscript. All authors reviewed and approved the final version of the manuscript.

\section{Ethics approval and consent to participate}

This study was approved by the Ethics Committee of the Jichi Medical University Hospital (A16-048). All procedures performed in the studies involving human participants were in accordance with the 1964 Helsinki declaration and its later amendments or similar ethical standards. The written informed consent from the patients enrolled was waived by the Ethics Committee because the plasma samples were obtained after routine laboratory tests.

\section{Consent for publication}

Not applicable in this study.

\section{Competing interests}

The authors declare that they have no competing interests.

\section{Publisher's Note}

Springer Nature remains neutral with regard to jurisdictional claims in published maps and institutional affiliations.

\begin{abstract}
Author details
'Department of Infectious Diseases, The University of Tokyo Hospital, 7-3-1 Hongo, Bunkyo-ku, Tokyo 113-8655, Japan. ${ }^{2}$ Division of General Internal Medicine, Jichi Medical University Hospital, 3311-1 Yakushiji, Shimotsuke-shi, Tochigi 329-0498, Japan. ${ }^{3}$ Department of Pharmacy, The University of Tokyo Hospital (Current affiliation: The Education Center for Clinical Pharmacy, Graduate School of Pharmaceutical Sciences, The University of Tokyo), 7-3-1 Hongo, Bunkyo-ku, Tokyo 113-8655, Japan. ${ }^{4}$ Department of Medical Informatics, Center for Information, Jichi Medical University, 3311-1 Yakushiji, Shimotsuke-shi, Tochigi 329-0498, Japan. ${ }^{5}$ Division of Infectious Diseases, Jichi Medical University Hospital, 3311-1 Yakushiji, Shimotsuke-shi, Tochigi 329-0498, Japan
\end{abstract}

Received: 29 August 2017 Accepted: 14 November 2017

Published online: 28 November 2017

\section{References}

1. Steenbergen JN, Alder J, Thorne GM, Tally FP. Daptomycin: a lipopeptide antibiotic for the treatment of serious gram-positive infections. J Antimicrob Chemother. 2005:55:283-8.

2. Liu C, Bayer A, Cosgrove SE, Daum RS, Fridkin SK, Gorwitz RJ, et al. Clinical practice guidelines by the Infectious Diseases Society of America for the treatment of methicillin-resistant Staphylococcus Aureus infections in adults and children. Clin Infect Dis. 2011;52:e18-55.

3. Webster PS, Oleson FB Jr, Paterson DL, Arkin CF, Mangili A, Craven DE, et al. Interaction of daptomycin with two recombinant thromboplastin reagents leads to falsely prolonged patient prothrombin time/international normalized ratio results. Blood Coagul Fibrinolysis. 2008;19:32-8.

4. van den Besselaar AMHP, Breukink E, Koorengevel MC. Phosphatidylglycerol and daptomycin synergistically inhibit tissue factor-induced coagulation in the prothrombin time test. J Thromb Haemost. 2010:8:1429-30.

5. Yamada T, Kato R, Oda K, Tanaka H, Suzuki K, ljiri Y, et al. False prolongation of prothrombin time in the presence of a high blood concentration of daptomycin. Basic Clin Pharmacol Toxicol. 2016;119:353-9.

6. Merck \& Co., Inc. (2017) Drug information: CUBICIN ${ }^{\circledR}$ (daptomycin for injection). https://www.merck.com/product/usa/pi_circulars/c/cubicin/ cubicin_pi.pdf. Accessed 19 May 2017

7. Saito M, Hatakeyama S, Hashimoto H, Suzuki T, Jubishi D, Kaneko M, et al. Dose-dependent artificial prolongation of prothrombin time by interaction between daptomycin and test reagents in patients receiving warfarin: a prospective in vivo clinical study. Ann Clin Microbiol Antimicrob. 2017;16:27.
8. Benvenuto M, Benziger DP, Yankelev S, Vigliani G. Pharmacokinetics and tolerability of daptomycin at doses up to 12 milligrams per kilogram of body weight once daily in healthy volunteers. Antimicrob Agents Chemother. 2006:50:3245-9.

9. Baumann Kreuziger LM, Kim B, Wieselthaler GM. Antithrombotic therapy for left ventricular assist devices in adults: a systematic review. J Thromb Haemost. 2015;13:946-55.

10. Whitlock RP, Sun JC, Fremes SE, Rubens FD, Teoh KH. Antithrombotic and thrombolytic therapy for valvular disease: antithrombotic therapy and prevention of thrombosis, 9th ed: American College of Chest Physicians Evidence-Based Clinical Practice Guidelines. Chest. 2012:141:e576S-600S.

\section{Submit your next manuscript to BioMed Central and we will help you at every step:}

- We accept pre-submission inquiries

- Our selector tool helps you to find the most relevant journal

- We provide round the clock customer support

- Convenient online submission

- Thorough peer review

- Inclusion in PubMed and all major indexing services

- Maximum visibility for your research

Submit your manuscript at www.biomedcentral.com/submit
) BioMed Central 